Saman Kermani

Parviz Ghadimi

http://dx.doi.org/10.21278/brod68407

ISSN 0007-215X

eISSN 1845-5859

\title{
ASSESSMENT OF FLOW INTERACTIONS BETWEEN CIRCULAR CYLINDERS AND NACA-0018 HYDROFOILS AT LOW REYNOLDS NUMBERS
}

UDC 629.572.:519.6

Original scientific paper

\section{Summary}

Force oscillations and flow around submerged objects at low Reynolds numbers are very important subjects in marine environments. On the other hand, NACA hydrofoils and circular sections are two of the most prevalent shapes that are applied in marine systems and due to the stated reason their hydrodynamic investigation becomes immensely important. Accordingly, in this paper, a flow solver has been developed based on finite volume method, and flow interactions between circular cylinders and NACA-0018 hydrofoils have been considered. For this purpose, different parametric studies have been conducted on the angles of attack and the distance of the foil from the cylinder at three different Reynolds numbers of 100, 200, and 300. Moreover, frequency of the lift and drag coefficients have been analyzed in different cases. As a result, suitable arrangements of the cylinders and foils are determined in the context of an energy conversion system where force fluctuation is helpful and flow around foils and cylinders where fluctuation of forces around them are harmful and vibration or noise propagation should be controlled.

Key words: Cylinder; NACA foil; Finite volume method; Vortex shedding; Low Reynolds

\section{Nomenclature:}

$\begin{array}{cccc}\mathbf{u} & \text { Velocity } & \mathbf{f} & \text { Frequency } \\ \mathbf{t} & \text { Time } & \mathbf{I} & \text { Characteristic Length } \\ \mathbf{p} & \text { Pressure } & v & \text { Kinematic Viscosity } \\ \mathbf{N}_{\mathbf{f}} & \text { Number of face } & \tilde{u} & \text { Interpolated Velocity } \\ \mathbf{x} & \text { Coordinate system } & \mathbf{S t} & \text { Strouhal number } \\ \mathbf{R e} & \text { Reynolds Number } & \mathbf{D} & \text { Diameter of Cylinder } \\ \mathbf{C}_{\mathbf{L}} & \text { Lift Coefficient } & \mathbf{A} & \text { Cell area } \\ \Delta l & \text { Length of Face } & \frac{\partial}{\partial n} & \text { Normal Derivative } \\ \mathbf{C}_{\mathbf{D}} & \text { Drag Coefficient } & \text { ADI } & \text { Alternating Direction Implicit }\end{array}$




$\begin{array}{cccc}\rho & \text { Density } & \text { RANS } & \text { Reynolds Average Navier Stokes } \\ \mathbf{S} & \text { Surface Area } & \text { LES } & \text { Large Eddy Simulation } \\ \mathbf{n}_{\mathbf{i}} & \text { Unit Normal vector } & \text { URANS } & \text { Unsteady Reynolds Average Navier } \\ \text { DNS } & \text { Direct Numerical Simulation } & & \text { Stokes }\end{array}$

\section{Introduction}

Circular and foil shapes objects have many applications in marine vehicles and structures such as submarines, ships, and bridges over rivers. Generally, flow around bodies at low Reynolds number exhibits various types of swinging. Meanwhile, hydrofoils are used in energy conversion systems in underwater conditions. In these systems, fluctuation of pressure on hydrofoils causes movement of hydrofoils. As a result, electricity can be produced from the relocations of hydrofoils. One of the ways of generating the fluctuation of pressure is to place a cylinder in front of the foil. This phenomenon leads to vibration and generation of different sounds which makes the investigation of the behavior of fluid and forces around these objects interesting, if not imperative. This task can be accomplished through experimental and numerical approaches. Many researches have been devoted to these types of investigations which are surveyed, next.

In 1960, Roshko [1] experimentally studied flow past a cylinder at very high Reynolds number and Strouhal and drag coefficients were investigated. Merrick and Bitsuamlak [2] investigated flow control around cylinders numerically and experimentally at high Reynolds number by changing the roughness of the surface.

Many researchers have also studied flow around cylinder, numerically. Fornberg [3] implemented new boundary condition at far field in the analysis of flow around cylinder. He used polar coordinate for the computational domain. Reynolds numbers considered in his analyses were under 300. Braza et al. [4] investigated the pressure and velocity distributions around cylinder at three Reynolds numbers up to 1000 at the near field. They applied ADI method to solve momentum and continuity equations. Liu et al. [5] also applied multi-grid methods to solve unsteady incompressible flow. They analyzed flow past cylinder for validation of their solver at Reynolds number of 200 and compared their results against several numerical and experimental data. Ding et al. [6] presented a new method for solving steady and unsteady Navier-Stokes equations. Their method was based on finite difference and meshless approach and they investigated flow around a cylinder at low Reynolds number for assessing their method. On the other hand, Meanwhile, Catalano et al. [7] investigated behavior of flow around cylinder by using two dimensional RANS and LES approach at high Reynolds number. Rahman et al. [8] also used finite volume approach to solve URANS equations. They studied flow passing a cylinder in laminar and turbulence regimes. Rajani et al. [9] analyzed vortex shedding of two and three dimensional circular cylinders in laminar flow. URANS equations were solved by applying finite volume scheme in these simulations. Kanaris et al. [10] also investigated flow around circular cylinder in a plane channel by two and three-dimensional DNS method. These analyses were conducted at low Reynolds numbers and vortex shedding was captured in different cases. Keramati et al. [11] studied flow past a cylinder numerically in laminar regime. They used finite volume method to solve navier stokes equations. On the other hand, Sato and Kobayashi [12] performed different analyses on wake flow of the current past a cylinder in laminar regime. For this purpose, they used Abaqus software to solve the governing equations, numerically. Selstad [13] studied flow past a circular cylinder in turbulent regime. For simulating this problem, STAR-CCM software was used. Also, LES method was employed for turbulence modeling. Ghadimi et al. [14] considered the effect of splitter plates on vortex shedding of a cylinder and probed into 
acoustic pressure at Reynolds numbers of 200. Finite element method was applied for their research.

Different experiments have also been conducted on flow around foil sections. $\mathrm{Hu}$ and Yang [15] conducted several experiments for investigating the flow separation near foils at low Reynolds number. Alam et al. [16] performed an experimental study on the behavior of flow near NACA 0012 foil at low Reynolds numbers. They considered wide range of angles of attack for different tests. Meanwhile, vortex shedding of different foils was studied by Yarusevych et al. [17], experimentally. They examined foils at three angles of attack and recorded pattern of the flow passing foils. On the other hand, specifications of flow around NACA 0018 were determined by Gim and Lee [18] experimentally. They demonstrated the form of tip vortex of this foil in several angles of attack.

Several papers have been presented which have examined flow around hydrofoils from various aspects. Sahin et al. [19] developed a fluid flow solver through DNS approach. The purpose of their code was to simulate flow separation around Eppler hydrofoils. Chung [20] also conducted a numerical study on the performance of rowing hydrofoils. He used a combined finite volume and cut cell approach to discretize the Navier-Stokes equations. Mateescu and Abdo [21] analyzed behavior of the fluid near the cambered hydrofoils at very low Reynolds numbers, numerically. Sparks [22] carried out some tests for the analyses of flow around corrugated foils at low Reynolds number. Ashraf et al. [23] considered the effects of velocity, thickness and camber on flapping NACA foils. They investigated performance of foils in propulsions systems and exported coefficients of pressure and thrust. In 2012, Mateescu et al. [24] analyzed flow around different hydrofoils near the wall boundary at low Reynolds numbers, numerically. For this purpose, they developed a Navier-Stokes solver based on the ADI scheme. Karim et al. [25] carried out simulation of NACA 0015 hydrofoil moving near the water surface. They applied combined RANS and VOF solver to analyze the generated surface wave from the foil. Also, $\mathrm{Xu}$ and $\mathrm{Wu}$ [26] used boundary element method for modeling the flow past an oscillatory hydrofoil. They specified the effects of periodic motion on lift and drag coefficients and Strouhal number.

As evident in the presented literature review, flow around foil, foil with flap, circular cylinder, and circular cylinder with splitter plate have been studied in different cited works. However, in the current paper, flow interaction between circular cylinders and foils are investigated at low Reynolds numbers. Accordingly, a flow solver is developed based on finite volume method and flow interactions between circular cylinders and NACA-0018 hydrofoils have been considered. Different parametric studies are conducted on the angles of attack and the distance between the foil and the cylinder at three different Reynolds numbers of 100, 200, and 300. Furthermore, frequency of the lift and drag coefficients are analyzed in different cases and suitable arrangements of the cylinder and foils are determined for the equipments based on the helpfulness or harmfulness of the force fluctuations around them.

\section{Governing Equations}

One of the most suitable methods for the analysis of flow field is solving the NavierStokes equations. These equations include momentum equations as

$$
\frac{\partial u_{i}}{\partial t}+\frac{\partial}{\partial x_{j}} u_{i} u_{j}=-\frac{\partial p}{\partial x_{i}}+\frac{1}{R e} \frac{\partial}{\partial x_{j}} \frac{\partial}{\partial x_{j}} u_{i}
$$

and continuity equation as

$$
\frac{\partial u_{i}}{\partial x_{i}}=0
$$


Velocity and pressure distributions are obtained by solving Equations 1 and 2. Subsequently, lift and drag coefficients can be computed from Equations 3 and 4, respectively.

$$
\begin{aligned}
& C_{L}=\frac{\text { Lift }}{0.5 \rho u^{2} S} \\
& C_{D}=\frac{\text { Drag }}{0.5 \rho u^{2} S}
\end{aligned}
$$

Here, $\rho$ is the density, $u$ is the velocity, $\mathrm{S}$ is the surface area, while $C_{L}$ and $C_{D}$ are Lift and Drag coefficients. Also, non-dimensional numbers of Reynolds and Strouhal are applied in this study which are defined as

$$
\begin{aligned}
& R e=\frac{u l}{v} \\
& S t=\frac{f l}{u}
\end{aligned}
$$

where $f$ is the frequency of vortex shedding, $l$ is the characteristic length and $v$ is the kinematic viscosity.

Discretization of the Navier-Stokes equations is outlined in the next section.

\section{Numerical Method}

As pointed out earlier, Navier-Stokes equations are the governing equations which are nonlinear. Pressure and velocity are coupled in N-S equations and different methods have been proposed to discretize them. In the current study, Finite Volume Method (FVM) is applied to solve the N-S equations and Fractional step method proposed by Kim and Choi [27] is adopted in order to achieve faster convergence for the velocity and pressure. Based on this numerical approach, Navier-Stokes equations are solved in four steps:

Step 1: Solving the momentum equations and calculating $\hat{u}_{i}$ by equation

$$
\frac{\hat{u}_{i}-u_{i}^{n}}{\Delta t}+\frac{1}{2} \frac{\partial}{\partial x_{j}}\left(\hat{u}_{i} u_{j}^{n}+u_{i}^{n} \hat{u}_{j}\right)=-\frac{\partial p^{n}}{\partial x_{i}}+\frac{1}{2} \frac{1}{\operatorname{Re}} \frac{\partial}{\partial x_{j}} \frac{\partial}{\partial x_{j}}\left(\hat{u}_{i}+u_{i}^{n}\right)
$$

Step 2: Correction of the velocity and computing $u^{*}$ from the relation

$$
\frac{u^{*}-\hat{u}_{i}}{\Delta t}=\frac{\partial p^{n}}{\partial x_{i}}
$$

Step 3: Solving the Poisson's equation for the new pressure $p^{n+1}$ in

$$
\frac{\partial}{\partial x_{i}} \frac{\partial p^{n+1}}{\partial x_{i}}=\frac{1}{\Delta t} \frac{\partial u_{i}^{*}}{\partial x_{i}}
$$

Step 4: Correcting the velocity and determining the $u_{i}^{n+1}$ by equation

$$
\frac{u_{i}^{n+1}-u_{i}^{*}}{\Delta t}=-\frac{\partial p^{n+1}}{\partial x_{i}}
$$

This procedure is applied to specify the velocity and pressure in every time step. 
For discretization of the above equations, integration must be performed over each cell. After integration and using the divergence theorem, these equations in summation form become

$$
\begin{gathered}
\frac{\hat{u}_{i}-u_{i}^{n}}{\Delta t}+\frac{1}{A} \sum_{k=1}^{N_{f}} \frac{1}{2}\left(\left(u_{i}^{n} n_{i}\right)\left(\hat{u}_{i}-u_{i}^{n}\right)+u_{i}^{n} n_{j}\left(\hat{u}_{j}-u_{j}^{n}\right)+2 u_{i}^{n}\left(u_{i}^{n} n_{i}\right)\right) \Delta l_{k} \\
=-\frac{1}{A} \sum_{k=1}^{N_{f}} p^{n} \cdot n_{i} \Delta l_{k}+\frac{1}{A} \sum_{k=1}^{N_{f}} \frac{1}{2} \frac{1}{R e} \frac{\partial}{\partial n}\left(\hat{u}_{i}+u_{i}^{n}\right) \Delta l_{k} \\
\frac{u^{*}-\hat{u}_{i}}{\Delta t}=\frac{1}{A} \sum_{k=1}^{N_{f}} p^{n} \cdot n_{i} \Delta l_{k} \\
\sum_{k=1}^{N_{f}} \frac{\partial p^{n+1}}{\partial n} \Delta l_{k}=\frac{1}{\Delta t} \sum_{k=1}^{N_{f}} \tilde{u}_{i}^{*} n_{i} \Delta l_{k} \\
\frac{u_{i}^{n+1}-u_{i}^{*}}{\Delta t}=\frac{1}{A} \sum_{k=1}^{N_{f}} p^{n+1} \cdot n_{i} \Delta l_{k}
\end{gathered}
$$

In these equations, $\mathrm{A}$ is the cell area, $N_{f}$ is the number of faces in each cell, $\Delta l$ is the length of each face, and $n_{i}$ is the unit normal vector of each face. Also, $\tilde{u}$ is the interpolated velocity which is obtained by the Rhie and Chow method. Meanwhile, $\frac{\partial}{\partial n}$ is the normal derivative on each cell face which is determined through an approach outlined in reference [27]. According to this reference, normal derivative for the unstructured mesh cell (Figure 1) is defined as follows:

$$
\left.\frac{\partial \phi}{\partial n}\right|_{O_{f}}=\frac{\phi_{2}-\phi_{1}}{d_{1}+d_{2}}-\frac{\phi_{B}-\phi_{A}}{\Delta s} \tan \alpha
$$

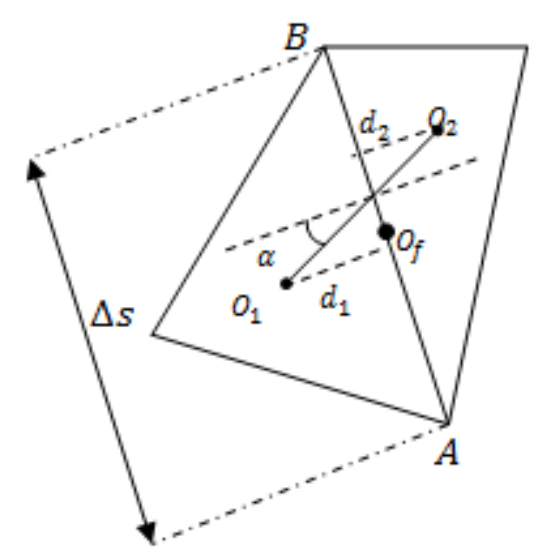

Figure 1: Schematics of two unstructured mesh cells.

In Equation 15, $O_{1}$ and $O_{2}$ are the centers of two adjacent mesh cells, $d_{1}$ is the normal distance from face $\mathrm{AB}$ to point $O_{1}, d_{2}$ is the normal distance from face $\mathrm{AB}$ to point $O_{2}, \alpha$ is the angle between the line $O_{1} O_{2}$ and the normal line to the face $\mathrm{AB}, \Delta s$ is the distance from $\mathrm{A}$ to B, while $O_{f}$ is the center of the face. 
A flow solver is hereby developed for computing the mentioned equations. The flow solver is written in C language and GNU-GCC compiler is applied for compiling and running the developed code. The prepared code was executed on a computer with an Intel core i7-870 CPU. The frequency of cores associated to this CPU is $2.93 \mathrm{GHz}$. Computational domain and problem setup are described in the next section.

\section{Problem Setup}

Computational domain and boundary conditions are illustrated in Figure 2. Diameter of the cylinder and chord length of the foil (NACA-0018) are designated to be D. Blockage ratio for all analyses is set to be $1 / 8$. Moreover, distance of the cylinder from the domain inlet is assumed to be 10D and distance of the cylinder from the domain outlet is assumed to be 20D. Boundary conditions of on the cylinder, foil, top and bottom of the domain are assumed to be no slip wall.

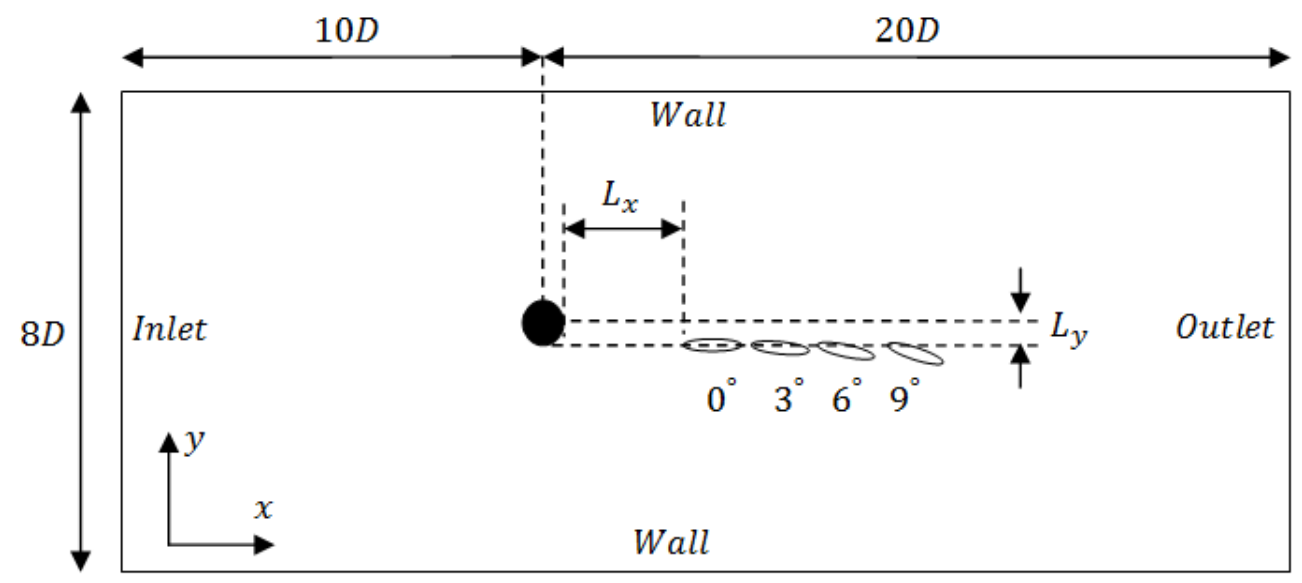

Figure 2: Illustration of computational domain and boundary conditions.

For investigating the flow interaction between circular cylinders and the hydrofoils, several analyses are considered. First, validation of the developed Navier-Stokes solver is performed through a lid driven cavity problem and flow around a cylinder at Reynolds number of 200. Subsequently, NACA-0018 hydrofoil is considered at six different positions and three angles of attack at the downstream of the cylinder which are listed in Table 1.

All of the intended analyses are carried out at three Reynolds numbers of 100, 200, and 300. In all the considered cases, $\mathrm{D}$ is assumed to be $1 \mathrm{~m}$ and the inlet velocities for the three Reynolds numbers of 100, 200 and 300 are $0.0001004 \mathrm{~m} / \mathrm{s}, 0.000208 \mathrm{~m} / \mathrm{s}$, and $0.0003012 \mathrm{~m} / \mathrm{s}$, respectively.

Table 1: Positions and angles of attack of NACA-0018.

\begin{tabular}{|c|c|c|c|}
\hline Number of Case & $L_{x}$ & $L_{y}$ & $\alpha$ (Degree) \\
\hline 1 & $D$ & 0 & 0 \\
\hline 2 & $3 D$ & 0 & 0 \\
\hline 3 & $5 D$ & 0 & 0 \\
\hline 4 & $D$ & $0.5 D$ & 0 \\
\hline 5 & $3 D$ & $0.5 D$ & 0 \\
\hline 6 & $5 D$ & $0.5 D$ & 0 \\
\hline
\end{tabular}




\begin{tabular}{|c|c|c|c|}
\hline 7 & $3 D$ & 0 & 3 \\
\hline 8 & $3 D$ & 0 & 6 \\
\hline 9 & $3 D$ & 0 & 9 \\
\hline 10 & $3 D$ & $0.5 D$ & 3 \\
\hline 11 & $3 D$ & $0.5 D$ & 6 \\
\hline 12 & $3 D$ & $0.5 D$ & 9 \\
\hline
\end{tabular}

\section{Results and Discussion}

\subsection{Validation}

Validation of the developed flow solver is accomplished based on a lid driven cavity problem at Reynolds number of 1000 and flow over a cylinder at Reynolds number of 200.

For the first validation case, a square computational domain with dimensions $1 \mathrm{~m}$ is assumed. Also, 10000 meshes are used to solve the cavity problem. Velocity field for the flow at Reynolds number of 1000 is displayed in Figure 3.

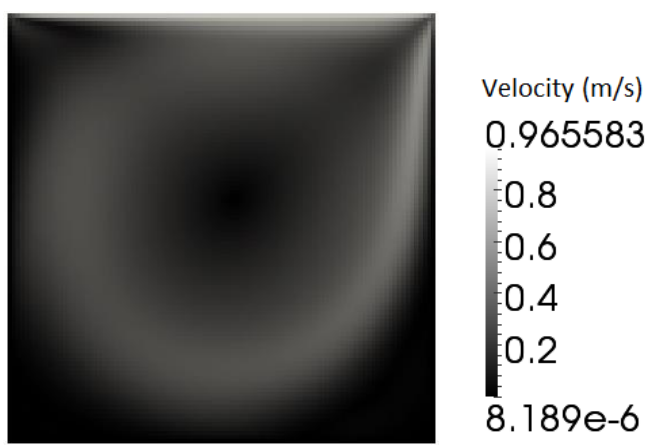

Figure 3: Velocity field of cavity problem at $\mathrm{Re}=1000$.

In addition, horizontal and vertical velocities at the vertical and horizontal center lines of the domain are illustrated in Figures 4 and 5. As evident in these Figure s, comparisons of the computed velocities against the results of Ghia [28] have displayed good agreements.

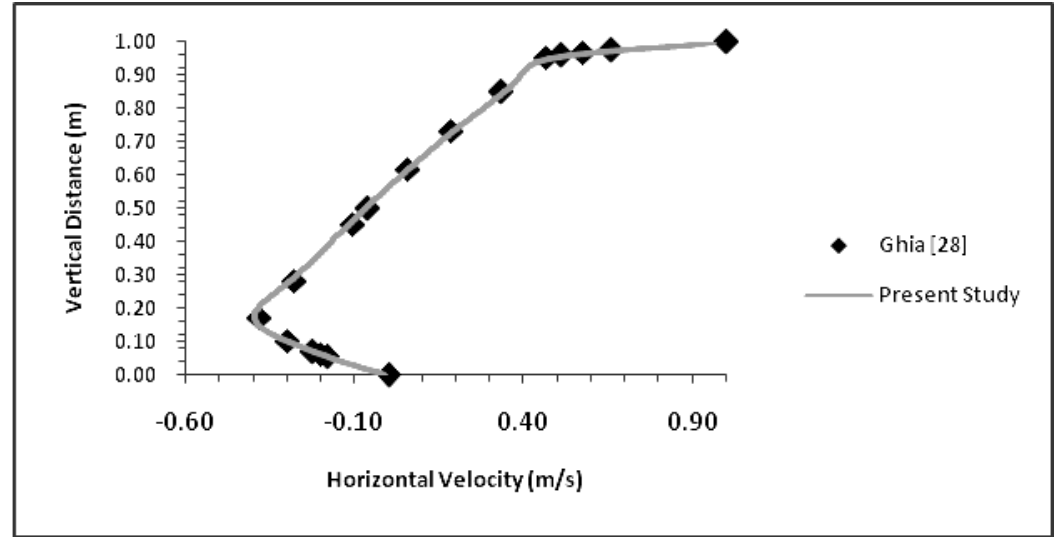

Figure 4: Horizontal velocity at the vertical center line. 


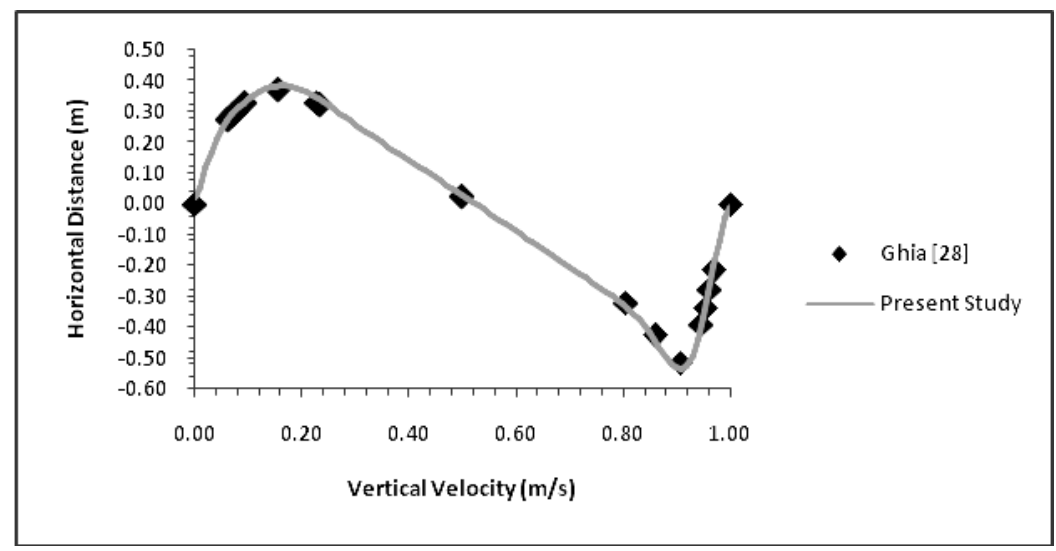

Figure 5: Vertical velocity at the horizontal center line.

For the second validation case, problem of flow over the circular cylinder at Reynolds number of 200 is adopted. To this end, gird independency analysis is conducted for six different meshes. Lift amplitudes versus number of grids are shown in Figure 6.

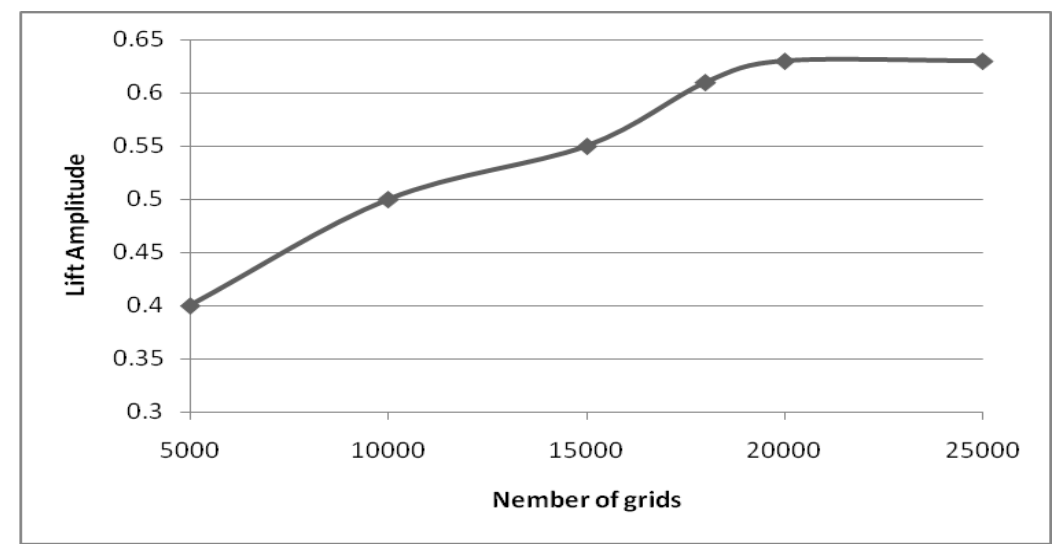

Figure 6: Lift amplitude versus number of grids.

Results of a mesh study illustrate that lift amplitude does not change when number of grids is more than 20000. Accordingly, number of the unstructured mesh is chosen to be 20000 for all other considered cases. The computational domain for the analysis of the flow over a cylinder with 20000 grids is demonstrated in Figure 7.

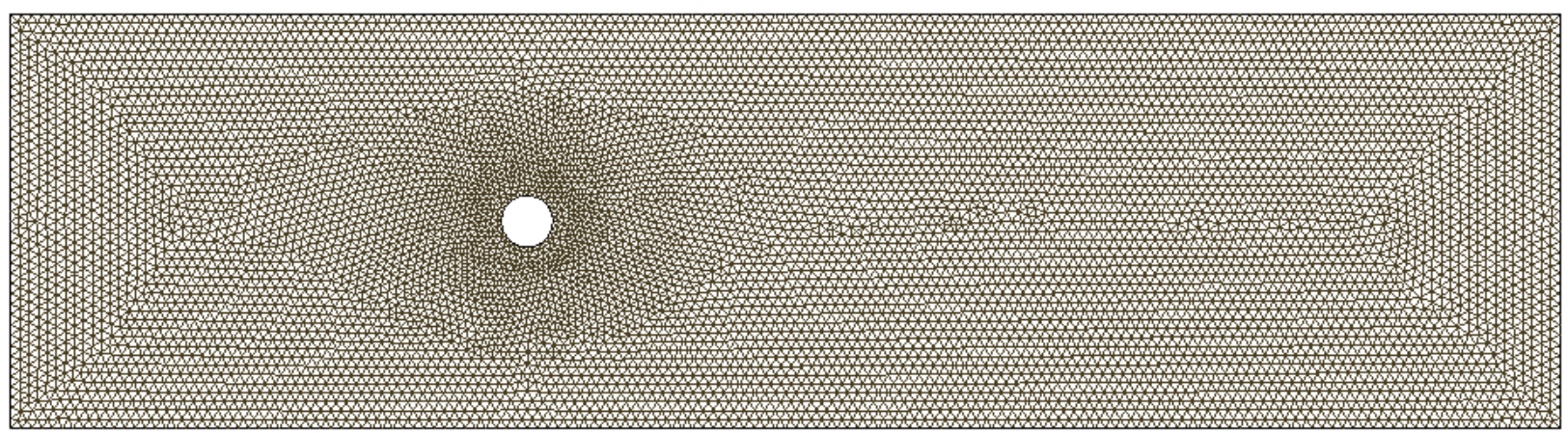

Figure 7: Unstructured mesh in the computational domain. 
The computed lift and drag coefficients at $\mathrm{Re}=200$ are displayed in Figures 8 and 9, respectively.

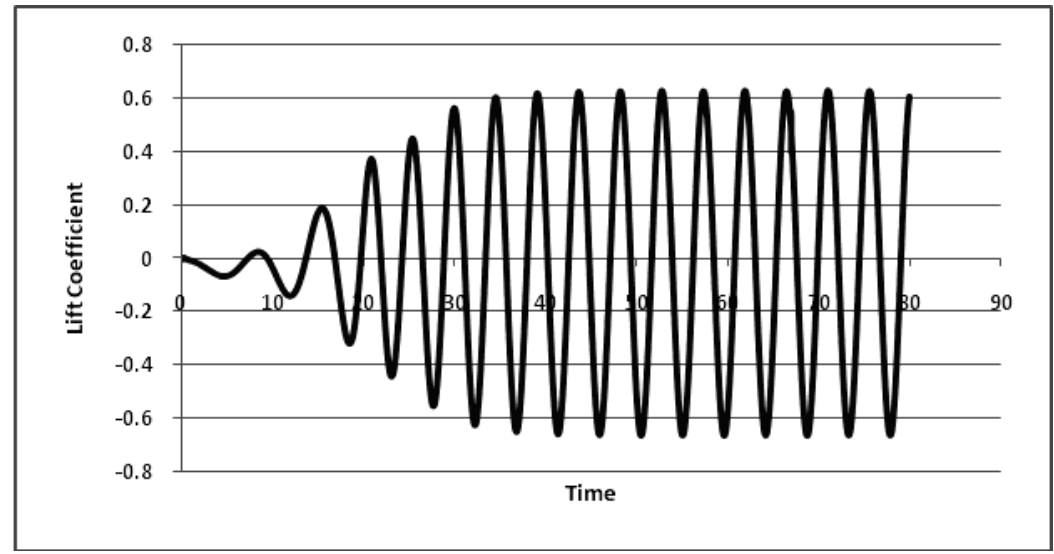

Figure 8: Lift coefficient of the cylinder at $\mathrm{Re}=200$.

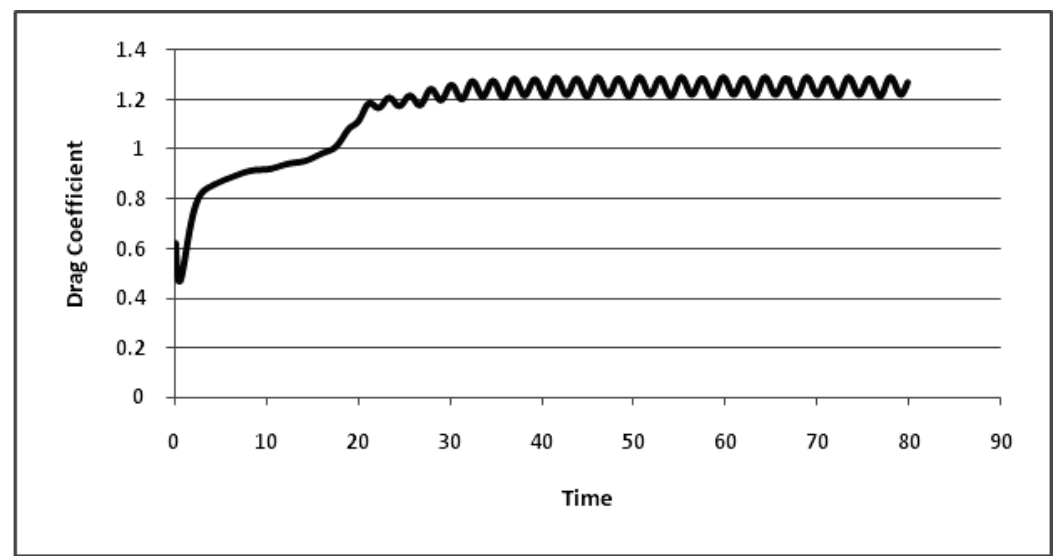

Figure 9: Drag coefficient of cylinder at $\mathrm{Re}=200$.

Plots of the drag and lift coefficients in Figures 8 and 9 show that results are steady after 40 seconds. The simulation of 40 seconds of the flow over a cylinder takes about 8 hours by the developed computer code. The computed lift and drag coefficients as well as the Strouhal number are compared against the results of two experimental and numerical studies in Table 2.

Table 2: Comparison of the results of the current study against experimental and numerical findings.

\begin{tabular}{|c|c|c|c|c|}
\hline Reference & Type & Amplitude of Lift & Average of Drag & Strouhal \\
\hline Present Work & Numerical & 0.63 & 1.25 & 0.217 \\
\hline Rogers et al. [29] & \multirow{3}{*}{ Numerical } & 0.65 & 1.23 & - \\
\cline { 1 - 3 } Error (\%) & & 3 & 1.6 & - \\
\hline Henderson [5] & \multirow{2}{*}{ Experimental } & - & - & 0.197 \\
\cline { 1 - 3 } Error (\%) & & - & - & 10.1 \\
\hline Vennard [30] & \multirow{2}{*}{ Experimental } & - & 1.24 & - \\
\cline { 1 - 4 } & & - & 0.8 & - \\
\hline
\end{tabular}


Based on the comparison presented in Table 2, maximum error of the solver is found to be approximately 10 percent. Based on this observation, one may conclude the accuracy of the developed flow solver in predicting pressure and velocity fields.

\subsection{Numerical Results}

In this section, flow interactions between NACA-0018 hydrofoils and circular sections are analyzed in 12 different foil cases which were described in Table 1 at three Reynolds numbers. Velocity fields of case 9 at different Reynolds numbers are demonstrated in form of contours in Figure 10. Based on these contour plots, it is clear that frequency increases as the Reynolds number increases.

a

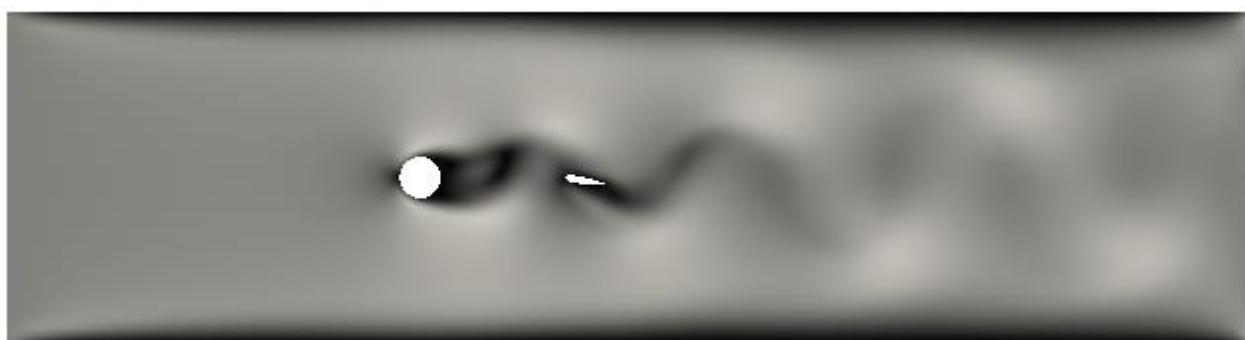

b
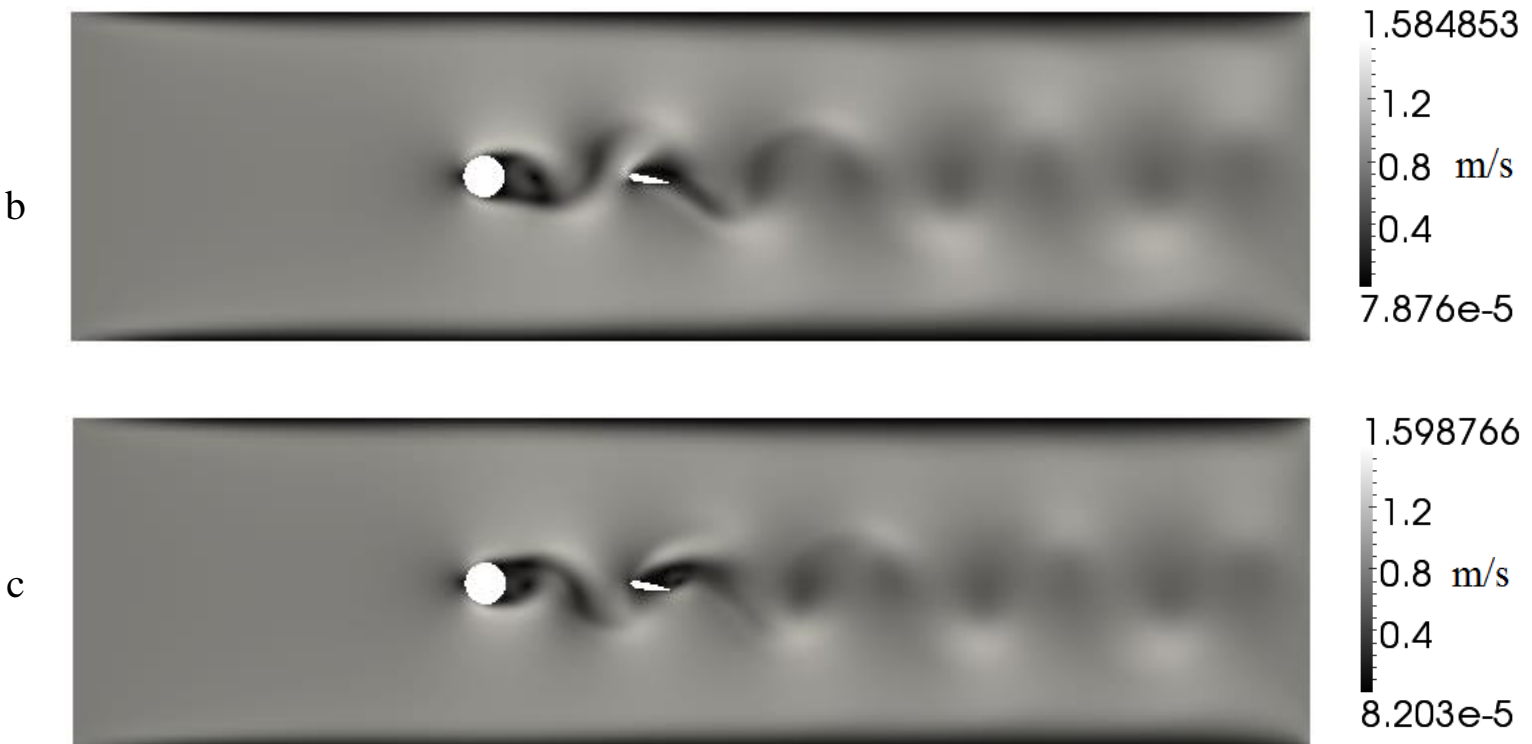

Figure 10: Velocity fields of case 9 at three Reynolds numbers at time $=100 \mathrm{~s}(\mathrm{a}: \mathrm{Re}=100, \mathrm{~b}: \mathrm{Re}=200, \mathrm{c}: \mathrm{Re}=300$ ).

As evident in Figure 10, all contours become steady in 100 seconds. Lift coefficients of the cylinder in case 9 are also shown in Figure 11. Based on these graphs, convergence is achieved sooner by higher Reynolds number. 

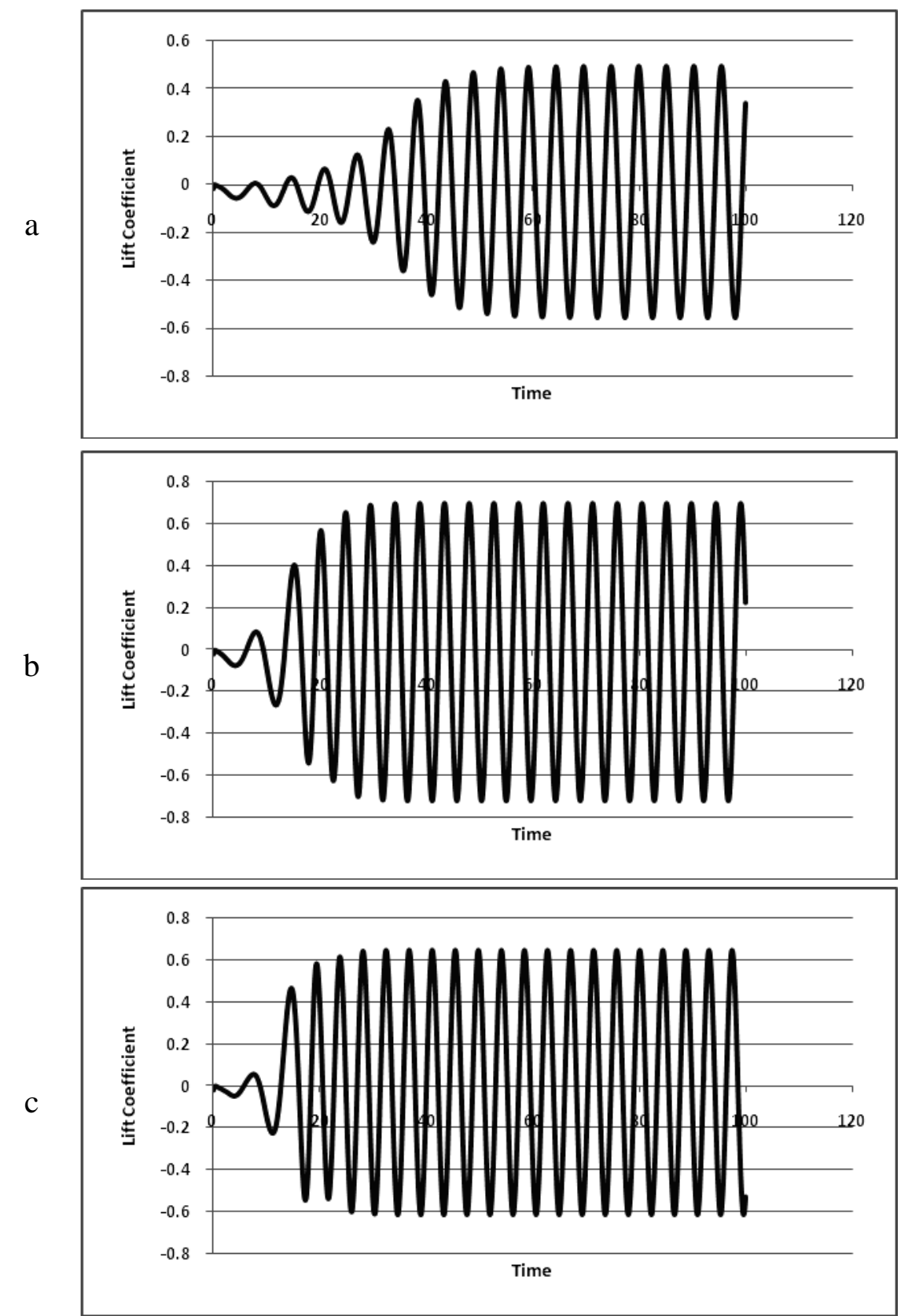

Figure 11: Lift coefficients of case 9 at three Reynolds numbers (a:Re=100, b:Re=200, c:Re=300).

Lift and drag coefficients as well as Strouhal number for NACA foil and cylinder in all cases are presented in Tables 3 and 4.

Table 3: Lift and drag coefficients as well as Strouhal number of cylinder for all cases (ave=Average, amp=Amplitude).

\begin{tabular}{|c|c|c|c|c|c|c|c|c|c|c|c|c|c|c|c|}
\hline \multirow{3}{*}{$\begin{array}{c}\text { Number } \\
1 \\
\end{array}$} & \multicolumn{5}{|c|}{$\mathrm{Re}=100$} & \multicolumn{5}{|c|}{$\mathrm{Re}=200$} & \multicolumn{5}{|c|}{$\mathrm{Re}=300$} \\
\hline & \multicolumn{2}{|c|}{$\mathrm{C}_{\mathrm{L}}(\mathrm{ave})(\mathrm{amp})$} & \multicolumn{2}{|c|}{$\begin{array}{l}\mathrm{C}_{\mathrm{D}} \text { (ave) } \\
\text { (amp) }\end{array}$} & \multirow{2}{*}{$\begin{array}{c}\mathrm{St} \\
0.152\end{array}$} & \multicolumn{2}{|c|}{$\mathrm{C}_{\mathrm{L}}(\mathrm{ave})(\mathrm{amp})$} & \multicolumn{2}{|c|}{$\begin{array}{c}\mathrm{C}_{\mathrm{D}} \text { (ave) } \\
\text { (amp) }\end{array}$} & \multirow{2}{*}{$\begin{array}{c}\text { St } \\
0.161\end{array}$} & \multicolumn{2}{|c|}{$\mathrm{C}_{\mathrm{L}}(\mathrm{ave})(\mathrm{amp})$} & \multicolumn{2}{|c|}{$\begin{array}{c}\mathrm{C}_{\mathrm{D}} \text { (ave) } \\
\text { (amp) }\end{array}$} & \multirow{2}{*}{$\begin{array}{c}\text { St } \\
0.161\end{array}$} \\
\hline & -0.013 & 0.058 & 1.225 & 0.000 & & -0.010 & 0.119 & 1.065 & 0.002 & & 0.005 & 0.100 & p.899 & 0.009 & \\
\hline 2 & -0.034 & 0.524 & 1.348 & 0.020 & 0.192 & -0.011 & 0.709 & 1.237 & 0.044 & 0.217 & 0.014 & 0.633 & 1.055 & 0.041 & 0.233 \\
\hline 3 & -0.035 & 0.437 & 1.359 & 0.014 & 0.192 & -0.019 & 0.612 & 1.236 & 0.034 & 0.217 & 0.008 & 0.591 & 1.062 & 0.036 & 0.233 \\
\hline 4 & -0.027 & 0.036 & 1.201 & 0.005 & 0.172 & -0.023 & 0.063 & 1.051 & 0.014 & 0.189 & 0.011 & 0.231 & p.925 & 0.038 & 0.175 \\
\hline 5 & -0.034 & 0.517 & 1.348 & 0.028 & 0.192 & -0.012 & 0.693 & 1.235 & 0.044 & 0.217 & 0.019 & 0.615 & 1.049 & 0.043 & 0.233 \\
\hline
\end{tabular}




\begin{tabular}{|c|c|c|c|c|c|c|c|c|c|c|c|c|c|c|c|}
\hline 6 & -0.037 & 0.438 & 1.359 & 0.014 & 0.192 & -0.022 & 0.611 & 1.236 & 0.035 & 0.217 & 0.012 & 0.586 & 1.060 & 0.036 & 0.233 \\
\hline 7 & -0.032 & 0.526 & 1.349 & 0.020 & 0.192 & -0.011 & 0.709 & 1.236 & 0.044 & 0.217 & 0.015 & 0.633 & 1.055 & 0.041 & 0.233 \\
\hline 8 & -0.031 & 0.526 & 1.347 & 0.023 & 0.192 & -0.011 & 0.710 & 1.235 & 0.044 & 0.217 & 0.016 & 0.633 & 1.054 & 0.042 & 0.233 \\
\hline 9 & -0.029 & 0.526 & 1.348 & 0.021 & 0.192 & -0.010 & 0.710 & 1.233 & 0.048 & 0.217 & 0.017 & 0.633 & 1.028 & 0.069 & 0.233 \\
\hline 10 & -0.032 & 0.515 & 1.352 & 0.024 & 0.192 & -0.011 & 0.690 & 1.235 & 0.044 & 0.217 & 0.020 & 0.613 & 1.050 & 0.043 & 0.233 \\
\hline 11 & -0.030 & 0.514 & 1.352 & 0.024 & 0.192 & -0.009 & 0.689 & 1.235 & 0.043 & 0.217 & 0.021 & 0.612 & 1.050 & 0.043 & 0.233 \\
\hline 12 & -0.028 & 0.516 & 1.354 & 0.023 & 0.192 & -0.011 & 0.686 & 1.236 & 0.043 & 0.217 & 0.046 & 0.635 & 1.077 & 0.070 & 0.233 \\
\hline
\end{tabular}

Table 4 Lift and drag coefficients of NACA-0018 for all cases (ave=Average, amp=Amplitude).

\begin{tabular}{|c|c|c|c|c|c|c|c|c|c|c|c|c|}
\cline { 2 - 14 } \multicolumn{1}{c|}{} & \multicolumn{4}{|c|}{$\mathrm{Re}=100$} & \multicolumn{4}{c|}{$\mathrm{Re}=200$} & \multicolumn{4}{c|}{$\mathrm{Re}=300$} \\
\hline Number & \multicolumn{1}{c}{$\mathrm{C}_{\mathrm{L}}(\mathrm{ave})(\mathrm{amp})$} & $\mathrm{C}_{\mathrm{D}}(\mathrm{ave})(\mathrm{amp})$ & $\mathrm{C}_{\mathrm{L}}(\mathrm{ave})(\mathrm{amp})$ & $\mathrm{C}_{\mathrm{D}}(\mathrm{ave})(\mathrm{amp})$ & $\mathrm{C}_{\mathrm{L}}(\mathrm{ave})(\mathrm{amp})$ & \multicolumn{2}{c|}{$\mathrm{C}_{\mathrm{D}}(\mathrm{ave})(\mathrm{amp})$} \\
\hline 1 & 0.000 & 0.189 & -0.036 & 0.001 & 0.001 & 0.519 & -0.050 & 0.011 & -0.002 & 0.532 & -0.051 & 0.011 \\
\hline 2 & 0.002 & 1.648 & 0.005 & 0.048 & -0.003 & 1.855 & -0.010 & 0.079 & -0.006 & 1.784 & -0.017 & 0.084 \\
\hline 3 & 0.000 & 1.526 & 0.059 & 0.039 & 0.000 & 1.660 & 0.020 & 0.072 & 0.000 & 1.603 & 0.007 & 0.083 \\
\hline 4 & 0.019 & 0.117 & 0.030 & 0.013 & 0.038 & 0.323 & 0.037 & 0.041 & 0.119 & 0.536 & 0.042 & 0.066 \\
\hline 5 & -0.083 & 1.377 & 0.032 & 0.102 & -0.252 & 1.627 & -0.001 & 0.126 & -0.258 & 1.569 & -0.009 & 0.129 \\
\hline 6 & -0.221 & 1.449 & 0.064 & 0.076 & -0.259 & 1.619 & 0.027 & 0.110 & -0.230 & 1.588 & 0.020 & 0.119 \\
\hline 7 & 0.098 & 1.650 & -0.015 & 0.089 & 0.072 & 1.862 & -0.039 & 0.122 & 0.055 & 1.785 & -0.041 & 0.129 \\
\hline 8 & 0.189 & 1.642 & -0.006 & 0.149 & 0.155 & 1.858 & -0.037 & 0.188 & 0.133 & 1.784 & -0.052 & 0.178 \\
\hline 9 & 0.279 & 1.635 & 0.022 & 0.219 & 0.228 & 1.860 & -0.012 & 0.269 & 0.199 & 1.795 & -0.034 & 0.258 \\
\hline 10 & 0.038 & 1.328 & 0.028 & 0.134 & -0.155 & 1.577 & -0.023 & 0.159 & -0.157 & 1.522 & -0.033 & 0.153 \\
\hline 11 & 0.166 & 1.277 & 0.045 & 0.169 & -0.034 & 1.521 & -0.013 & 0.206 & -0.027 & 1.466 & -0.026 & 0.187 \\
\hline 12 & 0.292 & 1.235 & 0.079 & 0.208 & 0.085 & 1.482 & 0.017 & 0.258 & 0.092 & 1.436 & 0.005 & 0.239 \\
\hline
\end{tabular}

As observed in Table 3, Strouhal number is decreased when longitudinal distance of the foil from the cylinder is equal to D. Likewise, when foil is situated at the center line of the cylinder, minimum Strouhal number is achieved. It is also seen that variation of the foil position in y direction has no effect on the mean value of the drag coefficient of cylinder at three Reynolds numbers, but this coefficient is reduced by about 10 percent when the foil gets closer to the cylinder, as shown in Figure 12.

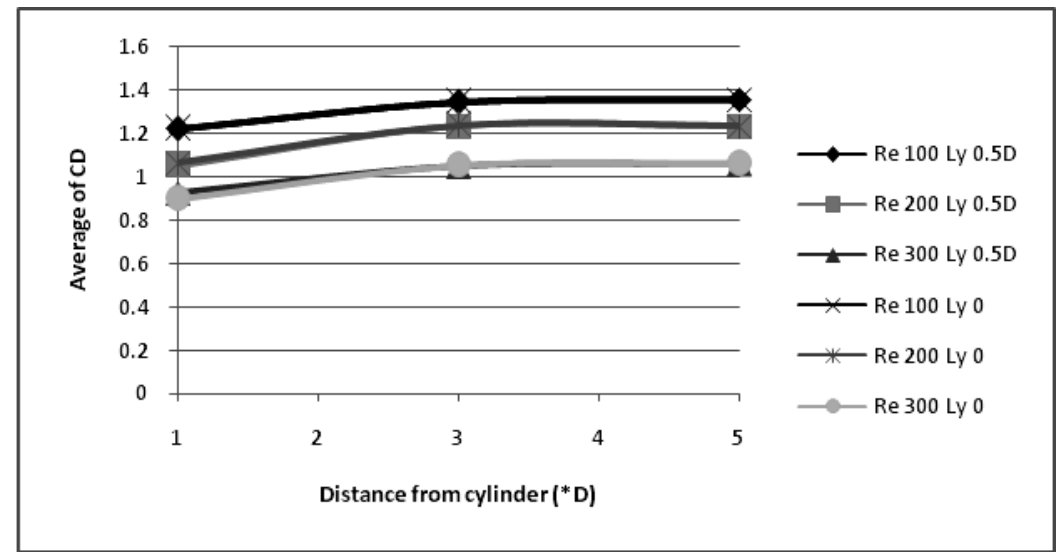

Figure 12: Average value of drag coefficients of the cylinder at different distances.

Variation of the amplitude of lift coefficient for the cylinder in cases 1 through 6 is illustrated in Figure 13. Maximum value of the amplitude of $C_{L}$ is seen at a longitudinal distance equal to 3D at all Reynolds numbers. Also, the amplitude at $\mathrm{Re}=200$ is found to be higher than the amplitudes at $\mathrm{Re}=100$ and $\mathrm{Re}=300$ by about $40 \%$ and $17 \%$, respectively. 


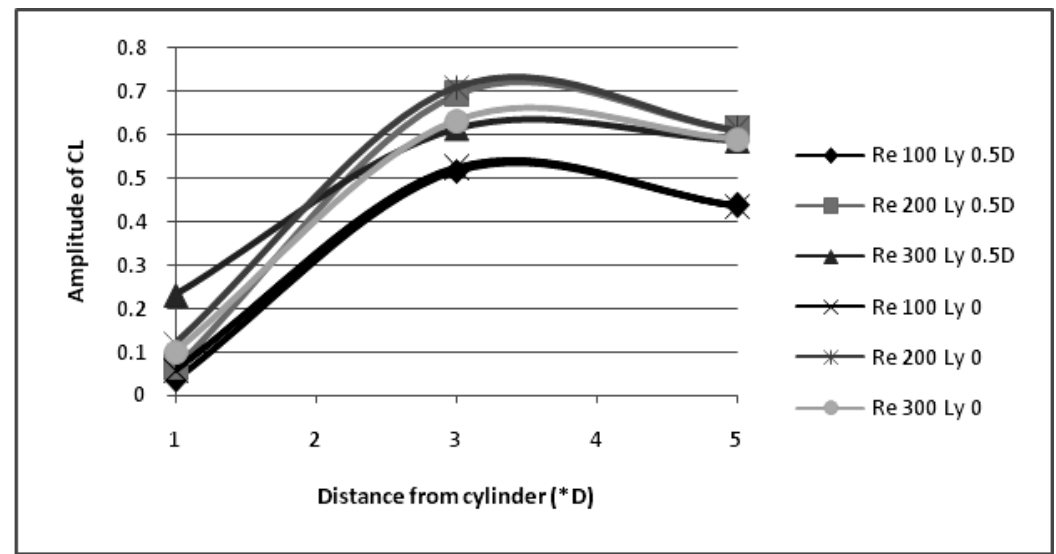

Figure 13: Amplitude of the lift coefficients of the cylinder at different distances.

Average value of the foil lift coefficients for cases 1 through 6 are displayed in Figure 14. In these cases, angle of attack is considered to be zero. Mean value of $C_{L}$ is about zero when the foil is situated at the center line of the cylinder, but sizable variation is observed when $\mathrm{L}_{\mathrm{y}}$ is equal to $0.5 \mathrm{D}$, while maximum absolute value is seen in case 5 at Reynolds number of 200.

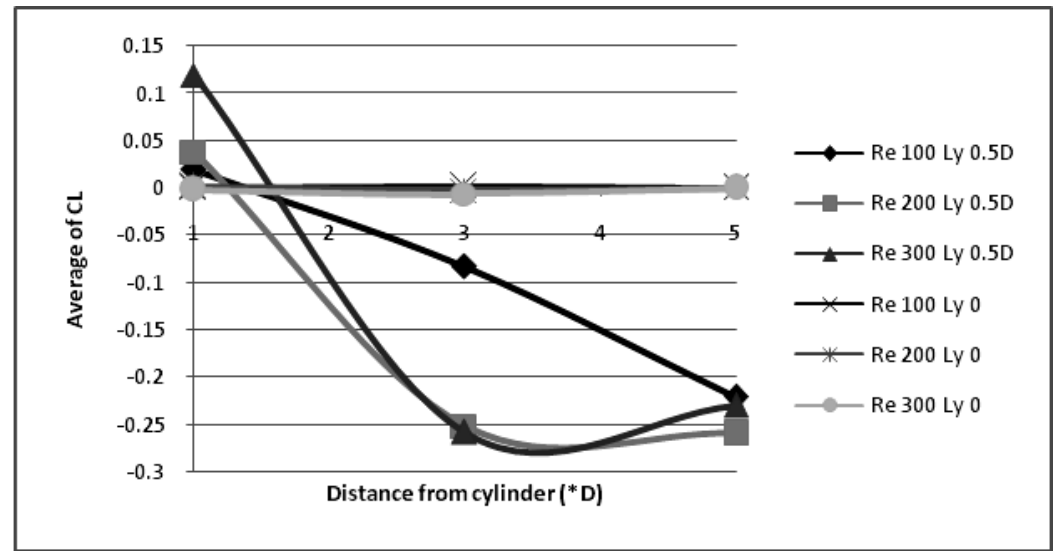

Figure 14: Average value of lift coefficients of the foil at different distances.

Amplitudes of $C_{L}$ and $C_{D}$ of the foil for zero angle of attack and different distances from the cylinder are illustrated in Figures 15 and 16, respectively. Amplitude of the lift coefficients for these cases displays same behavior. Maximum value of this parameter is seen at $\mathrm{Re}=200$ when the foil is placed along the center line of the cylinder, while minimum value occurs at $\mathrm{L}_{\mathrm{y}}=0.5 \mathrm{D}$ and $\mathrm{Re}=100$. Amplitudes of the drag coefficients display the same behavior, as well. Drags of the foils situated along the center line of the cylinder are greater than those in other positions by about 50 percent. Moreover, changes in the amplitude of the lift are 10 times more than changes in the amplitude of the drag. In other words, forces in $y$ direction are significantly greater than forces in $\mathrm{x}$ direction. 


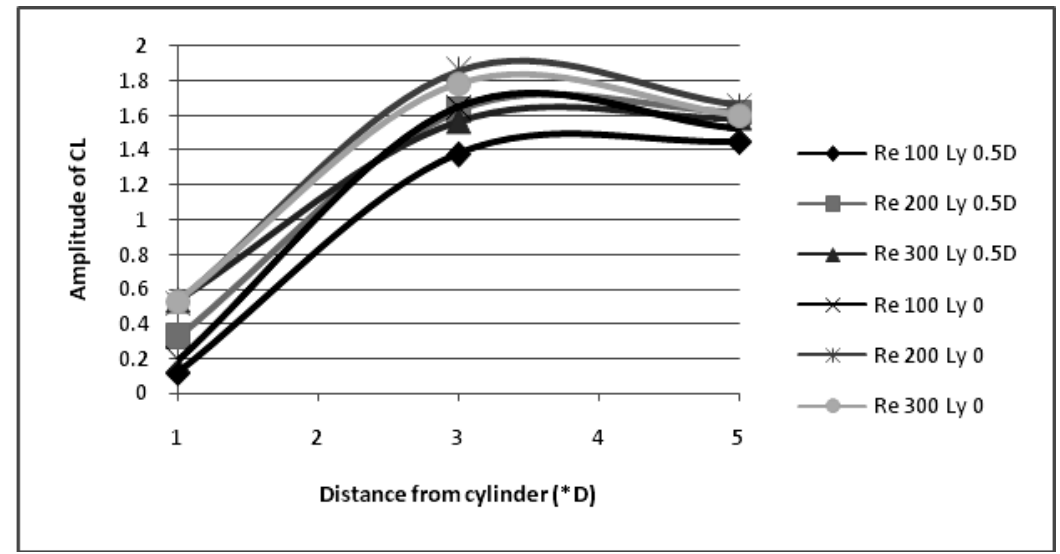

Figure 15: Amplitude of the lift coefficients of the foil at different distances.

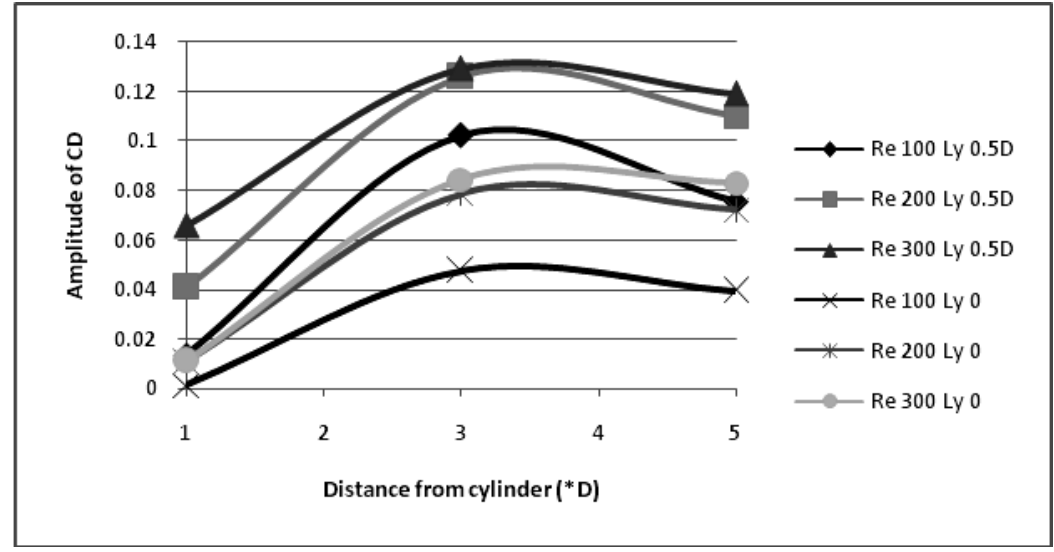

Figure 16: Amplitude of the drag coefficients of the foil at different distances.

Mean value of $C_{L}$ for different angles of attack are displayed in Figure 17. Lift coefficients of the foils situated along the center line of the cylinder, start from zero but coefficients of other foils start from a negative value because they are placed under the center line. At $\operatorname{Re}=100$,rate of growth of the coefficient for foils under the center line is higher than that of foils situated at the center line and their values are matched at 9 degrees angle of attack.

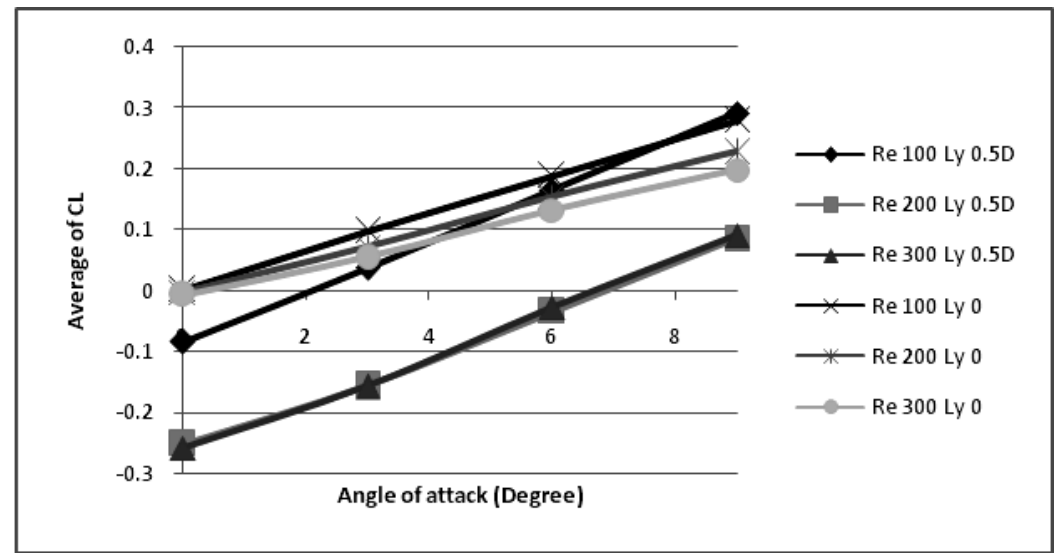

Figure 17: Average value of the lift coefficients of the foil at different angles of attack.

Amplitudes of $C_{L}$ and $C_{D}$ of the foils at different angles of attack are shown in Figure s 18 and 19, respectively. Increase in the angle of attack is seen to have no effect on the amplitude of $C_{L}$ when parameter $\mathrm{L}_{\mathrm{y}}$ for the foils is zero. On the other hand, amplitudes of lift 
coefficient are reduced about 0.2 by an increase in angle of attack for foils under the center line. In addition, maximum value of $C_{L}$ is seen at $\mathrm{Re}=200$ when the foil is placed on the center line. Minimum amplitude of the drag coefficients is seen to happen at $\operatorname{Re}=100$ and $\mathrm{L}_{\mathrm{y}}=0$. Also, Figure 18 illustrates that growth rate of the amplitude of $C_{D}$ for middle foils is more than that of foils under the center line. Moreover, maximum amplitude of the drag for different angles of attack is seen to occur at $\mathrm{Re}=200$.

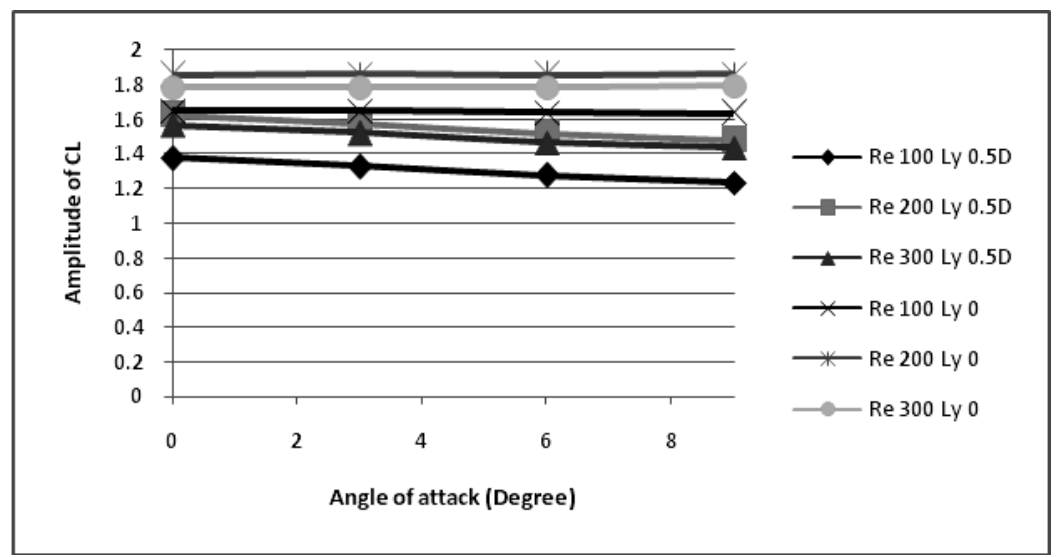

Figure 18: Amplitude of the lift coefficients of the foil at different angles of attack.

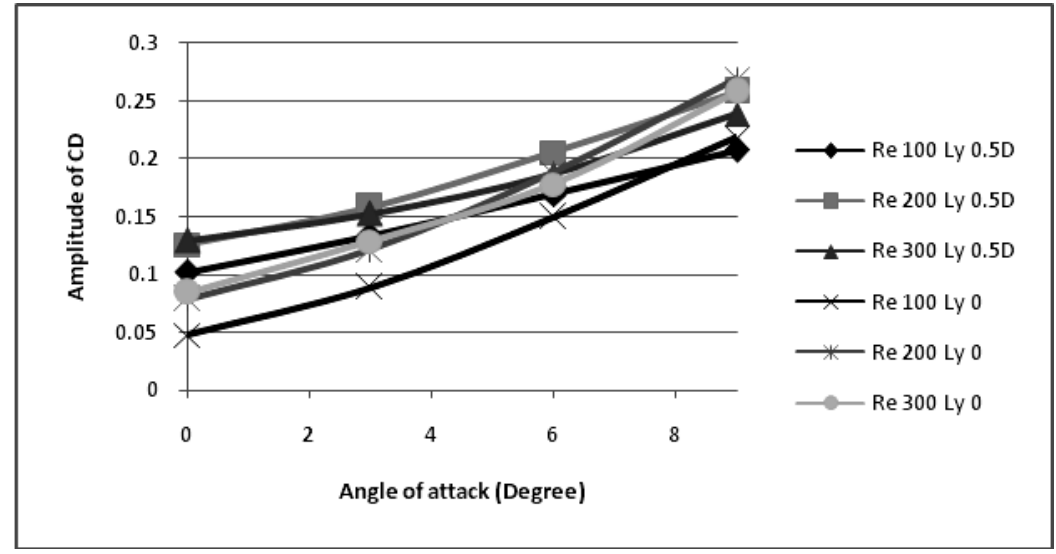

Figure 19: Amplitude of the drag coefficients of the foil at different angles of attack.

Generally, the drag frequency is twice of the frequency of the lift in some vortex shedding problems. However, in the considered cases, an increase in foil's angle of attack causes the frequency of $C_{D}$ reach the frequency of $C_{L}$ and an in increase in the drag coefficient. In other words, frequency of the drag coefficient is decreased by an increase in angle as a result of vortex shedding of the cylinder. This phenomenon is seen at $R e=200$ in four graphs of Figure 20.
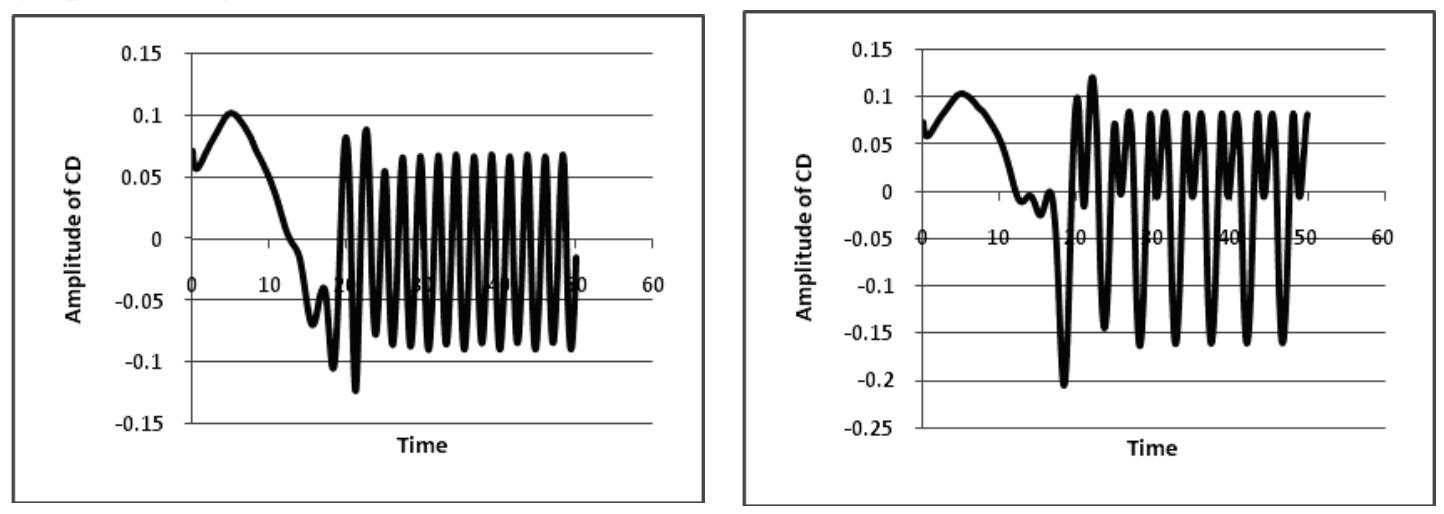
a

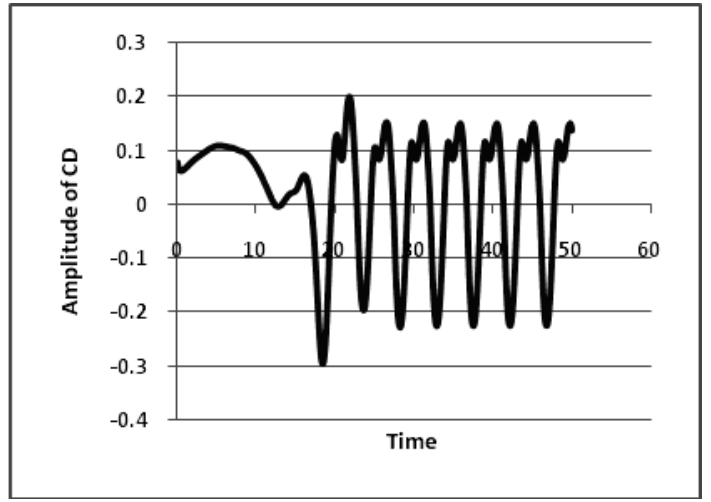

$\mathrm{c}$ $\mathrm{b}$

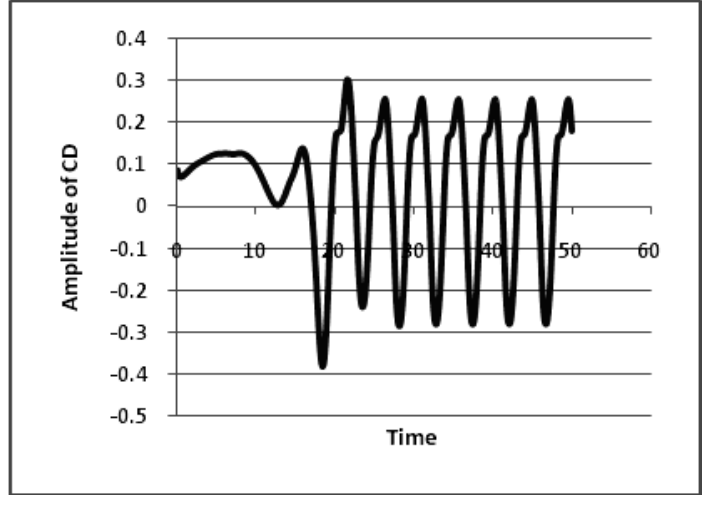

d

Figure 20: Time history of drag coefficients of the foil in four angles of attack (a:0 degree, b:3 degrees, c:6 degrees, d:9 degrees).

\section{Conclusions}

Flow interactions between NACA-0018 hydrofoils and circular sections are investigated and force oscillations around these profiles are studied. To achieve this goal, a Navier-stokes solver is developed through FVM approach. The developed code is validated by a lid driven cavity and flow over a cylinder. Subsequently, flow interaction between the mentioned objects is investigated for different angles of attack and several distances of the foil from the cylinder.

In some marine equipment such as energy conversion systems, fluctuation of force is helpful, while in some others like flow around foils or cylinders, force oscillations should be controlled in order to prevent vibration or sound propagations. Based on the results of the current study, suitable positions of a hydrofoil from a cylinder is determined for different low Reynolds numbers. For example, a distance $L_{x}=3 D$ at Reynolds number of 200 is found to be an appropriate position for an energy conversion system where the lift oscillation is at its maximum. On the other hand, minimum space from a foil to a cylinder is found to be suitable for equipments whose force fluctuations are harmful for them where the fluctuation of the lift and drag is at its minimum.

Complete analyses have also been carried out on the frequency of lift and drag amplitude of the cylinder and NACA-0018 hydrofoil in the current study. The obtained results indicate that frequency of the lift is decreased when the foil gets closer to the cylinder. Meanwhile, graphs of the drag coefficients of NACA-0018 foil at different angles of attack demonstrates that frequency of the drag could reach the frequency of the lift by an increase in the angle of attack. This phenomenon occurs for NACA-0018 foil under the effect of vortex shedding of the cylinder. 


\section{REFERENCES}

[1] Roshko, A. :Experiments on the flow past a circular cylinder at very high Reynolds number, Journal of Fluid Mechanics, Vol.10, 1961, pp. 345-356.

[2] Merrick, R., Bitsuamlak, G., :Control of flow around a circular cylinder by the use of surface roughness: A computational and experimental approach, Technical Report International Hurricane Research Center, 2009.

[3] Fornberg, B., :A numerical study of steady viscous flow past a circular cylinder, Journal of Fluid Mechanics, Vol.98, 1980, pp. 819-855.

[4] Braza, M., Chassaing, P., Ha Minh, H., :Numerical study and physical analysis of the pressure and velocity fields in the near wake of a circular cylinder, Journal of Fluid Mechanics, Vol.165, 1986, pp. 79-130.

[5] Liu, C., Zheng, X., Sung, C. H., :Preconditioned Multigrid Methods for Unsteady Incompressible Flows, Journal of Computational Physics, Vol.139, 1998, pp. 35-57.

[6] Ding, H., Shu, C., Yeo, K.S., Xu, D., :Simulation of incompressible viscous flows past a circular cylinder by hybrid FD scheme and meshless least square-based finite difference method:, Computer Methods in Applied Mechanics and Engineering., vol.193, 2003, pp. 727-744.

[7] Catalano, P., Wang, M., Iaccarino, G., Moin, P., :Numerical simulation of the flow around a circular cylinder at high Reynolds numbers, International Journal of Heat and Fluid Flow ,vol. 24, 2003, pp. 463-469.

[8] Rahman, M., Karim, M., Alim, A., :Numerical investigation of unsteady flow past a circular cylinder using 2-d finite volume method, Journal of Naval Architecture and Marine Engineering, vol.4, 2007, pp. $27-42$.

[9] Rajani, B.N., Kandasamy, A., Majumdar, S., :Numerical simulation of laminar flow past a circular cylinder", Applied Mathematical Modelling, vol.33, 2008, pp.1228-1247.

[10] Kanaris, N., Grigoriadis, D., Kassinos, S., :Three dimensional flow around a circular cylinder confined in a plane channel, Journal of Physics of Fluids, Vol. 23, 2011.

[11] Farhoud, R.K., Amiralaie, S., Jabbari, G., Amiralaie, S., :Numerical Study of Unsteady Laminar Flow around a Circular Cylinder, Journal of Civil Engineering and Urbanism, Vol. 2, 2012, pp.63-67.

[12] Sato, M., Kobayashi, T., :A fundamental study of the flow past a circular cylinder using Abaqus/CFD, SIMULIA Community Conference, 2012.

[13] Selstad, S. :Simulation Of Viscous Flow Around A Circular Cylinder With Star-Ccm, Master Thesis, Norwegian University Of Science And Technology, 2013.

[14] Ghadimi, P., Kermani, S., Razughi, S., Zamanian, R., :Aerodynamic and Acoustical Analysis of Flow around a Circular Cylinder in a Channel and Parametric Study on the Effects of a Splitter Plate on the Generated Vibration and Noise, Journal of Applied Mathematics and Physics, Vol. 1, 2013, pp.1-5.

[15] Hu, H., Yang, Z., :An Experimental Study of the Laminar Flow Separation on a Low-Reynolds-Number Airfoil, Journal Of Fluids Engineering, Vol. 130, 2008.

[16] Alam, M., Zhou, Y., Yang, H.X., Guo, H., Mi, J., :The ultra-low Reynolds number airfoil wake, Experiments in Fluids, Vol. 48, 2009, pp.81-103.

[17] Yarusevych, S., Sullivan, P.E., Kawall, J.G., :On vortex shedding from an airfoil in low-Reynolds-number flows, Journal of Fluid Mechanics, Vol. 632, 2009, pp. 245-271.

[18] Gim, O.S., Lee, G.W., :Flow characteristics and tip vortex formation around a NACA 0018 foil with an endplate, Ocean Engineering, Vol. 60, 2012, pp.28-38.

[19] Sahin, M., Hall, J., Mohseni, K., :Direct Numerical Simulation of Separated Low-Reynolds Number Flows around an Eppler 387 Airfoil, 46th AIAA Aerospace Sciences Meeting and Exhibit, 2008.

[20] Chung, M.H., :Numerical study of rowing hydrofoil performance at low Reynolds numbers, Journal of Fluids and Structures, Vol. 24, 2008, pp.313-335.

[21] Mateescu, D., Abdo, M., :Analysis of flows past airfoils at very low Reynolds numbers, Journal of Aerospace Engineering, Vol. 224, 2009, pp.757-775.

[22] Sparks, J.R., :Experimental Flow Visualization For Corrugated Airfoils At Low Reynolds Number Including Development Of A Pitch And Plunge Fixture, Master's Theses, University of Kentucky, 2011.

[23] M. A. Ashraf, J. Young and J.C.S. Lai, "Reynolds number, thickness and camber effects on flapping airfoil propulsion", Journal of Fluids and Structures, Vol. 27, 2011, pp.145-160. 
[24] Mateescu, D., Scholz, O., Wang, C., :Aerodynamics Of Airfoils At Low Reynolds Numbers In The Proximity Of The Ground, 12th Pan-American Congress of Applied Mechanics, 2011.

[25] Karim, M., Prasad, B., Rahman, N., :Numerical simulation of free surface water wave for the flow around NACA 0015 hydrofoil using the volume of fluid (VOF) method, Ocean Engineering, Vol. 78, 2013, pp.89-94.

[26] Xu, G.D., Wu, G.X., :Boundary element simulation of inviscid flow around an oscillatory foil with vortex sheet, Journal of Engineering Analysis with Boundary Elements, Vol. 37, 2013, pp.825-835.

[27] Kim, D., Choi, H., :A Second-Order Time-Accurate Finite Volume Method for Unsteady Incompressible Flow on Hybrid Unstructured Grids, Journal of Computational Physics, Vol. 162, 2000, pp.411-428.

[28] Ghia, U., Ghia, KN., Shin, CT., :High-Re solution for incompressible flow using the Navier-Stokes equations and a multigrid method, Journal of Computational Physics, Vol. 48, 1982, pp.387-411.

[29] Rogers, S.E., Kwak, D., :Upwind differencing scheme for the time-accurate incompressible Navier-Stokes equations, Journal of AIAA, Vol. 28, 1990.

[30] Vennard, J.K., :Elementary Fluid Mechanics, John Wiley \& Sons Publication, New York, 1940, pp.311.

Submitted: $\quad$ 15.07. 2015. Saman Kermani

Accepted: $\quad$ 03.03.2016. Department of Marine Technology, Amirkabir University of Technology, Tehran, Iran

Tell: (+9821) 64543120; Fax: (+9821) 66412495 